\title{
Phytohormone and Light Regulation of Chlorophyll Degradation
}

\author{
Xiaoyu Zhu ${ }^{1,2 \dagger}$, Junyi Chen ${ }^{1,2 \dagger}$, Kai Qiu ${ }^{1,2 t}$ and Benke Kuai ${ }^{1,2 \star}$ \\ ${ }^{1}$ State Key Laboratory of Genetic Engineering and Fudan Center for Genetic Diversity and Designing Agriculture, School of \\ Life Sciences, Fudan University, Shanghai, China, ${ }^{2}$ Ministry of Education, Key Laboratory for Biodiversity Science and \\ Ecological Engineering, Institute of Biodiversity Science, Fudan University, Shanghai, China
}

\section{OPEN ACCESS}

Edited by:

Benoit Schoefs,

University of Maine, France

Reviewed by:

Carole Anne Llewellyn

Swansea University, United Kingdom Avtar Krishan Handa,

Purdue University, United States

*Correspondence:

Benke Kuai

bkkuai@fudan.edu.cn

${ }^{\dagger}$ These authors have contributed equally to this work.

Specialty section:

This article was submitted to Plant Physiology,

a section of the journal

Frontiers in Plant Science

Received: 14 August 2017 Accepted: 23 October 2017 Published: 06 November 2017

Citation:

Zhu X, Chen J, Qiu K and Kuai B (2017) Phytohormone and Light Regulation of Chlorophyll Degradation.

Front. Plant Sci. 8:1911.

doi: 10.3389/fp/s.2017.01911
Degreening, due to the net loss of chlorophyll (Chl), is the most prominent symptom during the processes of leaf senescence, fruit ripening, and seed maturation. Over the last decade or so, extensive identifications of Chl catabolic genes (CCGs) have led to the revelation of the biochemical pathway of Chl degradation. As such, exploration of the regulatory mechanism of the degreening process is greatly facilitated. During the past few years, substantial progress has been made in elucidating the regulation of Chl degradation, particularly via the mediation of major phytohormones' signaling. Intriguingly, ethylene and abscisic acid's signaling have been demonstrated to interweave with light signaling in mediating the regulation of Chl degradation. In this review, we briefly summarize this progress, with an effort on providing a framework for further investigation of multifaceted and hierarchical regulations of Chl degradation.

Keywords: chlorophyll degradation, phytohormone, ethylene, abscisic acid, jasmonic acid, light

\section{INTRODUCTION}

Chlorophyll (Chl) molecules are synthesized almost instantly upon light exposure of seedlings for harvesting light energy to drive photosynthesis in green organs, and during the processes of leaf senescence, fruit ripening, and seed maturation, they are degraded rapidly, a process called degreening, to facilitate nutrient remobilization and, in some cases, vitamin biosynthesis (Christ and Hörtensteiner, 2014; Vom Dorp et al., 2015). Chl degradation is in fact imperative to plant development for its detoxifying the photo-toxicity of Chl molecules once they are freed from their binding proteins (Hörtensteiner, 2006; Li et al., 2017). Over the last decade or so, the major biochemical pathway of $\mathrm{Chl}$ degradation has been revealed by cloning and function analysis of Chl catabolic genes (CCGs). Because of an important role of the pheophorbide $a$ oxygenase (PAO) in Chl degradation, this pathway is designated as PAO pathway (Christ and Hörtensteiner, 2014; Figure 1).

In higher plants, there are two forms of Chl molecules, $\mathrm{Chl} a$ and $\mathrm{Chl} b$. Chl $a$ is the degradable form of Chls, and, during leaf senescence, $\mathrm{Chl} b$ is converted to Chl $a$ by $\mathrm{Chl} b$ reductase [CBR, encoded by NON-YELLOW COLORING 1 (NYC1) and NYC1-LIKE (NOL)] and 7-hydroxymethyl Chl $a$ reductase (HCAR) (Kusaba et al., 2007; Horie et al., 2009; Sato et al., 2009; Meguro et al., 2011). For Chl $a$ degradation, Magnesium is initially removed to convert Chl $a$ to pheophytin $a$ (Phein $a$ ) by Magnesium-dechelatase, encoded by Mendel's green cotyledon genes, NON-YELLOWINGs/STAY-GREENs (NYEs/SGRs) (Armstead et al., 2007; Ren et al., 2007; Chen et al., 2016; Shimoda et al., 2016; Wu et al., 2016). Phein $a$ is then hydrolyzed by pheophytinase (PPH) to produce pheophorbide $a$ (Pheide $a$ ) and phytol (Morita et al., 2009; Schelbert et al., 2009; Ren et al., 2010). Remarkably, the green color of Chl catabolites is completely lost when the porphyrin ring of Pheide $a$ is cleaved by PAO, resulting in oxidized red Chl catabolite (RCC), which 


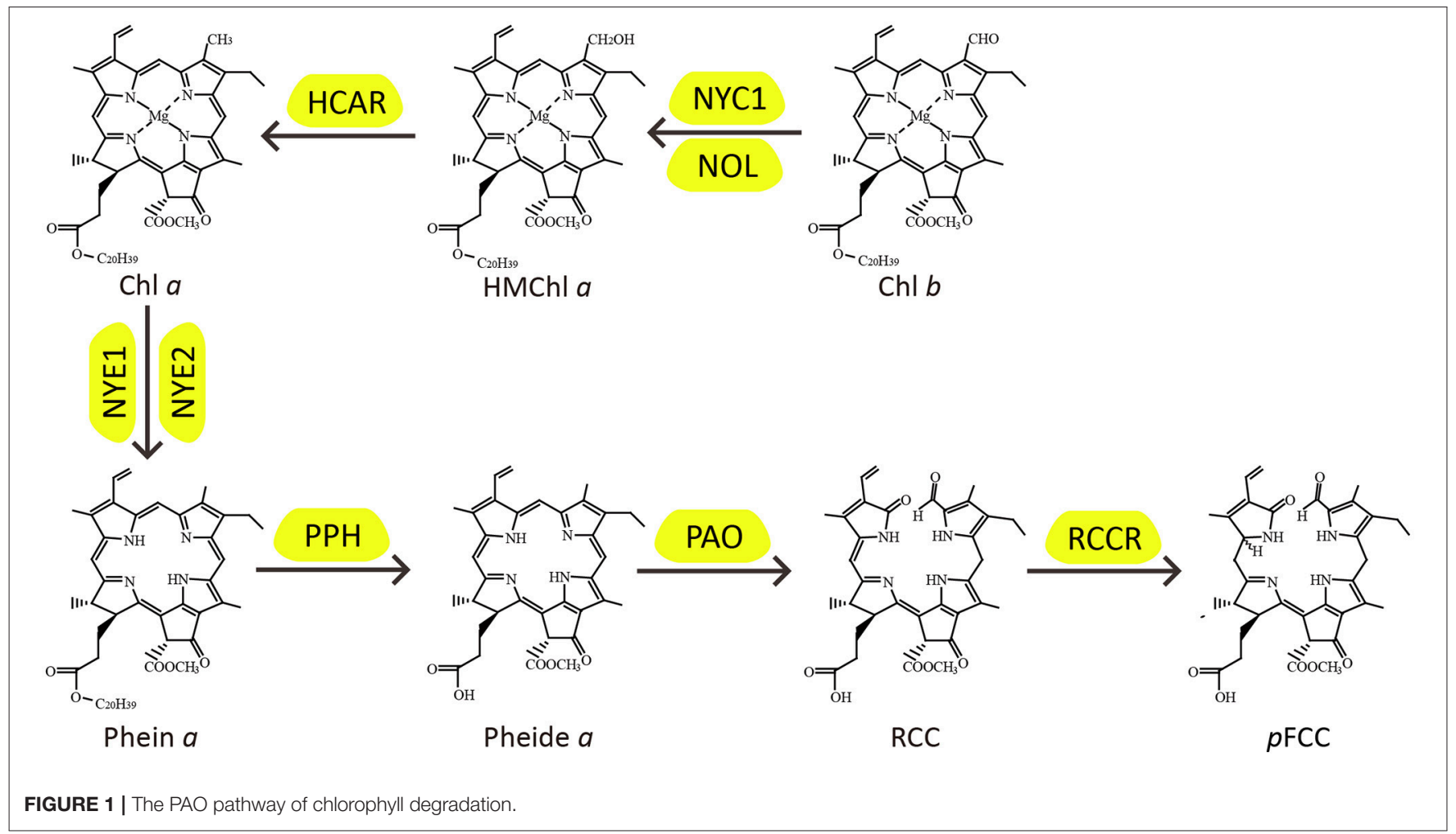

is subsequently catalyzed by red Chl catabolite reductase (RCCR) to generate primary fluorescent $\mathrm{Chl}$ catabolite $(p \mathrm{FCC}$ ) (Wüthrich et al., 2000; Pružinská et al., 2003; Pruzinská et al., 2007; Tanaka et al., 2003; Yao and Greenberg, 2006). Finally, the $p$ FCC is modified and transported into the vacuole, and isomerized to non-fluorescent products by acidic pH (Christ et al., 2012, 2013; Hauenstein et al., 2016).

Phytohormones and environmental factors have long been known to regulate Chl degradation (Lim et al., 2007); however, the molecular mechanisms involved in these regulations remains largely unknown. In last few years, the success in revealing the biochemical pathway of Chl degradation has led to a rapid progress in elucidation of the molecular mechanisms. Particularly, substantial progress has been made on elucidation of the regulatory roles of ethylene, abscisic acid (ABA), jasmonic acid (JA), and light signaling components on Chl degradation, and a number of regulatory factors of CCGs have been identified by using the methods of biochemistry, genetics, and bioinformatics (Delmas et al., 2013; Liang et al., 2014; Sakuraba et al., 2014, 2016; Song et al., 2014; Qiu et al., 2015; Zhang et al., 2015; Zhu et al., 2015; Gao et al., 2016; Ghandchi et al., 2016; Li et al., 2016; Oda-Yamamizo et al., 2016; Yin et al., 2016; Chen et al., 2017; Mao et al., 2017; Table 1). These advances provide some valuable insight into the complexity of the molecular mechanism of hormone- and light-regulated $\mathrm{Chl}$ degradation. Here, we review recent progress in this field and discuss important yet unresolved questions regarding the roles and mechanisms of phytohormones and environmental factors in Chl degradation regulation.

\section{THE MOLECULAR MECHANISM OF ETHYLENE SIGNALING-MEDIATED CHL DEGRADATION}

Ethylene is an important phytohormone, regulating diverse aspects of plant growth and development, especially leaf degreening and fruit ripening (Burg, 1973; Grbic and Bleecker, 1995; Lim et al., 2007; Qiu et al., 2015; Yin et al., 2016). During leaf degreening, the expression of ethylene biosynthetic genes encoding 1-Aminocyclopropane-1-carboxylic acid (ACC) synthase (ACS) and ACC oxidase (ACO) were significantly up-regulated, and the endogenous ethylene level increased accordingly (van der Graaff et al., 2006; Breeze et al., 2011). ACO1 antisense tomato plants synthesized less ethylene and delayed leaf degreening (John et al., 1995). ACSs octuple mutant, producing $\sim 10 \%$ of ethylene in WT, significantly delayed leaf degreening in Arabidopsis (Tsuchisaka et al., 2009). Exogenous application of ethylene could induce leaf degreening, whereas treatment with ethylene inhibitors could delay leaf degreening (Serek et al., 1995; Jing et al., 2005). The leaves of etr1-1, the mutant of ethylene receptor gene ETR1, cannot respond to ethylene treatment and shows a stay-green leaf phenotype (Bleecker et al., 1988; Grbic and Bleecker, 1995; Chao et al., 1997). Consistently, ectopic expression of a mutant form of the Arabidopsis ethylene receptor gene ETR1-1 delayed leaf Chl degradation in Nicotiana tabacum (Yang et al., 2008). ETHYLENE INSENSITIVE 2 (EIN2) and its downstream target EIN3 are key components of ethylene signaling, and the mutants of both EIN2 and EIN3 exhibit a severe stay-green phenotype 
TABLE 1 | The direct regulatory factors of Chl catabolic genes (CCGs).

\begin{tabular}{|c|c|c|c|c|c|c|}
\hline Species & Accession numbers & Regulatory factors & Signaling & Phenotypes of mutants & Target CCGs & References \\
\hline Arabidopsis thaliana & At3g20770 & EIN3 & Ethylene & Stay-green during leaf senescence & NYC1, NYE1, PAO & Qiu et al., 2015 \\
\hline Arabidopsis thaliana & At5g39610 & ORE1 & Ethylene & Stay-green during leaf senescence & $\begin{array}{l}\text { NYC1, NOL, NYE1, } \\
\text { PAO }\end{array}$ & Qiu et al., 2015 \\
\hline Citrus sinensis & Ciclev10010348m & CitERF13 ${ }^{a}$ & Ethylene & NA & CitPPH & Yin et al., 2016 \\
\hline Arabidopsis thaliana & At1g34180 & ANAC016 & Abscisic acid & Stay-green during leaf senescence & NYE1 & Sakuraba et al., 2016 \\
\hline Arabidopsis thaliana & At3g24650 & $\mathrm{ABI} 3$ & Abscisic acid & Stay-green during seed maturation & NYE1, NYE2 & Delmas et al., 2013 \\
\hline Arabidopsis thaliana & At1g16540 & $\mathrm{ABI} 5$ & Abscisic acid & Stay-green during leaf senescence & NYC1, NYE1 & Sakuraba et al., 2014 \\
\hline Arabidopsis thaliana & At1g30230 & EEL & Abscisic acid & Stay-green during leaf senescence & NYC1, NYE1 & Sakuraba et al., 2014 \\
\hline Oryza sativa & Os03g0327800 & OsNAPb & Abscisic acid & $\begin{array}{l}\text { Accelerated yellowing during leaf } \\
\text { senescence }^{\mathrm{C}}\end{array}$ & $\begin{array}{l}\text { OsSGR, OsNYC1, } \\
\text { OsNYC3, } \\
\text { OsRCCR1 }\end{array}$ & Liang et al., 2014 \\
\hline Arabidopsis thaliana & $\begin{array}{l}\text { At1g52890/ At3g15500/ } \\
\text { At4g27410 }\end{array}$ & ANAC019/055/072 ${ }^{f}$ & Jasmonic acid & Stay-green during leaf senescence & NYC1, NYE1, NYE2 & Zhu et al., 2015 \\
\hline Arabidopsis thaliana & At2g43010 & PIF4 & Light & Stay-green during leaf senescence & NYE1 & Song et al., 2014 \\
\hline Arabidopsis thaliana & At3g59060 & PIF5 & Light & Stay-green during leaf senescence & NYE1, NYC1 & Zhang et al., 2015 \\
\hline Arabidopsis thaliana & At2g45660 & SOC19 & Light & $\begin{array}{l}\text { Accelerated yellowing during leaf } \\
\text { senescence }\end{array}$ & NYE1, PPH & Chen et al., 2017 \\
\hline
\end{tabular}

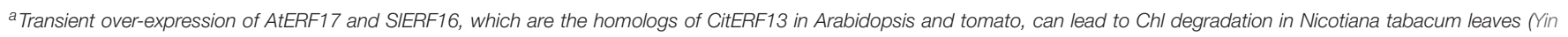
et al., 2016).

${ }^{b}$ The null mutant of AtNAP has a significant stay-green phenotype during leaf and silique senescence (Guo and Gan, 2006; Kou et al., 2012).

${ }^{c}$ The prematurely senile 1 (Ps1-D) is a gain-of-function mutant of OSNAP (Liang et al., 2014).

'OsNAC2 is a rice ortholog of ORE1/ANAC092/AtNAC2 (Mao et al., 2017).

e Over-expression of OsMYC2 significantly promote Chl degradation during leaf senescence in rice (Uji et al., 2017).

${ }^{f}$ Transient over-expression of oilseed rape BnaNAC55 (Brassica napus L.) lead to a significant decrease in Chl content in Nicotiana benthamiana leaves (Niu et al., 2016).

gSOC1 is a negative regulator of Chl degradation during leaf degreening and senescence (Chen et al., 2017).

during leaf senescence (Chao et al., 1997; Oh et al., 1997). EIN3 positively regulates $O R E 1$ and $N A P$, the two important regulatory genes of senescence, either directly or indirectly via negatively regulating miR164, which in turn cleaves the transcript of ORE1 (Kim et al., 2009, 2014; Li et al., 2013). These reports convincingly demonstrate that ethylene signaling regulates the pathway of $\mathrm{Chl}$ degradation.

Recently, Qiu et al. (2015) reported that the expression of NYC1, NYE1, and PAO was significantly induced by ethylene treatment in the leaves of Arabidopsis, whereas largely repressed in ein 3 eil1 double mutant. The electrophoretic mobility shift assay (EMSA) and dual-luciferase reporter assay demonstrated that EIN3 protein could directly bind to the EBS (EIN3 binding site, AC/TGA/TAC/TCT) in the promoters of NYC1, NYE1, and $P A O$, and enhance their promoter activity in Arabidopsis protoplasts. Therefore, EIN3 is a positive regulator of ethylene-mediated Chl degradation. Moreover, ORE1, the direct target of EIN3, could bind to the promoters of NYE1, NYC1, NOL, and PAO, and positively regulate their expression. Intriguingly, EIN3 and ORE1 could promote NYE1 and NYC1 expression in an additive manner (Qiu et al., 2015). This progress indicates that EIN3 and EIL1 constitute a major regulatory node of ethylene-triggered degreening, with EIN3 either directly or indirectly regulating the expression of CCGs. Notably, Yin et al. (2016) recently revealed that CitERF13, an ethylene responsive factor, could bind to $\mathrm{CitPPH}$ promoter and positively regulate its expression during citrus fruit degreening (Table 1).

\section{THE MOLECULAR MECHANISM OF ABA SIGNALING-MEDIATED CHL DEGRADATION}

ABA can be induced by age-dependent senescence or environmental stresses, such as drought, heat, and salt, and the increase of endogenous $\mathrm{ABA}$ level or the exogenous application of ABA accelerates chlorosis and senescence of plant leaves (Raab et al., 2009; Yang et al., 2014; Takasaki et al., 2015; Liu et al., 2016). ABA has therefore long been recognized as a positive regulator of degreening during leaf senescence in plants. It was reported that $\mathrm{ABA}$ accelerates leaf degreening and senescence via an AtNAP-SAG113 (a PP2C family protein phosphatase) regulatory module that is involved in the regulation of the stomata movement (Zhang and Gan, 2012).

With an attempt of investigating the direct regulation of CCGs, Gao et al. (2016) initially identified ABF3 as a transcriptional regulator of NYE1 by yeast one-hybrid (Y1H) screening. Further in vitro and in vivo analyses indicated that $\mathrm{ABF} 2 / 3 / 4$ directly bind to the promoter of NYE1, and 
up-regulate its transcription. Notably, $A B F 2 / 3 / 4$ also bind to the promoters of NYE2, NYC1, and $P A O$, and up-regulate their transcription. Under ABA treatment, detached leaves of abf2 abf3 abf4 triple mutants exhibited an obvious staygreen phenotype, while those of ABF4-OE transgenic lines showed an accelerated yellowing phenotype (Gao et al., 2016). ABI5 and EEL, two ABA signaling-related transcription factors, were also found to positively regulate the transcription of NYE1 and NYC1 by binding to their promoters (Sakuraba et al., 2014). Similarly, ANAC016, a senescence-associated NAC transcription factor, directly bind to the promoter of NYE1 and up-regulate its transcription. Leaves of anac016 mutant showed a stay-green phenotype, while ANAC016-OX line displayed an early leaf yellowing phenotype. Interestingly, it indirectly activates ABSCISIC ALDEHYDE OXIDASE3 (AAO3), an ABA biosynthesis gene, via a mediation of NAP (Kim et al., 2013; Yang et al., 2014; Sakuraba et al., 2016). Liang et al. (2014) found that ABA-induced leaf yellowing and senescence were mediated by OsNAP in rice. Unlike AtNAP, OsNAP was specifically induced by ABA but not ethylene. OsNAP directly bind to the promoters of OsSGR, OsNYC1, OsNYC3 (PPH), and $O s R C C R 1$, and up-regulated their transcription in rice. Recently, Mao et al. (2017) reported that OsNAC2 could directly bind to the promoters of OsSGR and OsNYC3, and activate their expression during $\mathrm{ABA}$-induced leaf yellowing and senescence in rice.

ABA also regulates seed maturation. During the processes of seed maturation and embryo degreening, a B3 domain transcription factor $\mathrm{ABI} 3$ directly binds to the promoters of NYE1 and NYE2, and up-regulates their transcription, consequently promoting $\mathrm{Chl}$ degradation in embryos. Intriguingly, the role of $\mathrm{ABI} 3$ in $\mathrm{Chl}$ degradation is seedspecific, as the mutant of $A B I 3$ (abi3-6) does not show a stay-green leaf phenotype in the dark (Delmas et al., 2013). This progress has shed a light on the complex molecular mechanism underlying ABA-regulated Chl degradation (Table 1).

\section{THE MOLECULAR MECHANISM OF JA SIGNALING-MEDIATED CHL DEGRADATION}

Jasmonic acid is a phytohormone essential for the regulation of multiple developmental processes, including leaf degreening and senescence (Wasternack and Hause, 2013). Ueda and Kato (1980) firstly found that methyl jasmonate (MeJA) could induce leaf degreening in oats. Subsequently, this phenomenon was confirmed in various plant species such as Arabidopsis, wheat, rice, and maize (Beltrano et al., 1998; He et al., 2002; Shan et al., 2011; Yan et al., 2012; Lee et al., 2015). Mutants defective for JA synthesis exhibited delayed leaf degreening phenotype (Castillo and León, 2008; Schommer et al., 2008; Yan et al., 2012). COI1-JAZ complex is the co-receptor of JA (Sheard et al., 2010), and the leaves of coil mutant exhibit a stay green phenotype upon MeJA treatment (He et al., 2002; Shan et al., 2011; Lee et al., 2015). MYC2/3/4 could interact with JAZ, acting as the transcriptional activators in JA signaling, whereas bHLH03/13/14/17 were identified as the transcriptional repressors, repressing JA responses. Both MYC2/3/4 and bHLH03/13/14/17 could bind to the promoter of $S A G 29$, and activate or repress the expression of SAG29 during JA-induced leaf senescence (Qi et al., 2015).

In a study of identifying the transcriptional regulators of CCGs, Zhu et al. (2015) revealed MYC2 as a putative transregulator of $P A O$ by using the $\mathrm{Y} 1 \mathrm{H}$ screening. MYC2 and its two homologs, MYC3 and MYC4, were then demonstrated to directly bind to the G-box (CACGTG) in the promoters of $P A O, N Y C 1$, and NYE1, and up-regulate their expression during JA-induced Chl degradation. The leaves of myc2 myc3 myc4 triple mutant showed a stay-green phenotype, whereas those of $\mathrm{MYC} 2 / 3 / 4$ overexpression lines displayed an accelerated yellowing phenotype upon MeJA treatment. Intriguingly, ANAC019/055/072, the immediate targets of MYC2/3/4 (Bu et al., 2008; Zheng et al., 2012), could also directly up-regulate the expression of NYE1, NYE2, and NYC1. The triple mutant of anac019 anac055 anac072 showed a similar stay-green phenotype as $m y c 2$ myc3 myc4 upon MeJA treatment. Moreover, the MYC2 and ANAC019 could interact with each other, and synergistically enhance NYE1 expression in Arabidopsis protoplasts. These findings indicate a hierarchical and coordinated regulatory network during JA-induced Chl degradation (Zhu et al., 2015; Table 1).

\section{THE MOLECULAR MECHANISM OF LIGHT SIGNALING IN REGULATING CHL DEGRADATION}

Light is the vital environmental factor for plant growth and development. Dark treatment, a simple and effective way for light deprivation, is widely used for studying leaf senescence and degreening (Ren et al., 2007; Christ and Hörtensteiner, 2014). phyB is a red light receptor (Schäfer and Bowler, 2002), and seedlings or mature leaves of $p h y B$ mutant yellow faster, whereas PHYB-OX plants yellow slower than those of WT during dark incubation (Sakuraba et al., 2014). phyB represses PIF4 and PIF5 at the post-transcriptional level (Leivar et al., 2008; Shin et al., 2009). In the dark, leaves of pif4, pif5, and pif1 pif3 pif4 pif5 quadruple mutants all show stay-green phenotypes, while those of PIF4-OX and PIF5-OX lines show early-yellowing phenotypes (Sakuraba et al., 2014). ELF3 inhibits leaf degreening and senescence by repressing PIF4 and PIF5 at the transcriptional level (Nusinow et al., 2011; Sakuraba et al., 2014). After incubating in darkness, leaves of elf3 senesced faster and leaves of ELF3-OX senesced slower than those of WT (Sakuraba et al., 2014). These findings collectively suggest that red light signaling is involved in the regulation of leaf degreening and senescence, with PIF4 and PIF5 acting as key mediators.

Both PIF4 and PIF5 associate with the promoters of $A B I 5$ and $E E L$, two bZIP family transcription factors, and up-regulate their transcription (Sakuraba et al., 2014). Interestingly, PIF4, PIF5, ABI5, and EEL, as well as EIN3, can all activate the 
expression of ORE1, which encodes an important senescencepromoting transcription factor, by directly binding to its promoter. Meanwhile, ABI5 and EEL could directly activate NYE1 and NYC1 by binding to their promoters (Sakuraba et al., 2014). It was further demonstrated that PIF4 directly bind to the promoter of NYE1, and PIF5 to the promoters of NYE1 and NYC1 to up-regulate their transcription (Song et al., 2014; Zhang et al., 2015). Under dark treatment, endogenous ethylene level was significantly reduced in the leaves of pif 4 mutant, while elevated in those of PIF4-OX lines. When treated with ethylene, mutants of pif3, pif4, and pif5 showed stay-green phenotypes, suggesting that PIF3/4/5 play roles in leaf degreening mediated by ethylene signaling (Song et al., 2014).

Recently, in a study designed for exploring the transcriptional regulation of $P P H$, Chen et al. (2017) demonstrated that SUPPRESSOR OF OVEREXPRESSION OF CO 1 (SOC1), a flowering pathway integrator, associates with the promoter of $P P H$, and negatively regulates its transcription. Under dark treatment, leaves of soc1-6 mutant yellowed earlier, whereas those of iSOC1-OE lines partially stayed green, in comparison to their respective controls. Moreover, SOC1 also negatively regulates NYE1 and SAG113 at the transcriptional level during dark-induced leaf degreening and senescence. Notably, SOC1 is the only negative regulator of Chl degradation identified so far (Table 1).

\section{CONCLUSION AND PERSPECTIVES}

Chl degradation is an active and progressive process which is regulated by diverse developmental and environmental clues, and mainly mediated by phytohormones' signaling. In Arabidopsis, ethylene signaling promotes leaf degreening through the transcriptional regulation of major CCGs by both EIN3 and ORE1, while in citrus fruits by CitERF13 (Qiu et al., 2015; Yin et al., 2016). The severe stay-green phenotype of the mutants of both EIN3/EIL1 and ORE1 implies that ethylene signaling is likely the major signaling pathway in regulating degreening during developmental leaf senescence (Kim et al., 2009; Li et al., 2013). ABA signaling mediates Chl degradation at the transcriptional level mainly by $\mathrm{ABI} 3$ during seed maturation, whereas, during leaf senescence, by ABI5, EEL, and ABF2/3/4 as well as ANAC016 in Arabidopsis, and by OsNAP and OsNAC2 in rice (Delmas et al., 2013; Liang et al., 2014; Sakuraba et al., 2014, 2016; Gao et al., 2016; Mao et al., 2017). Interestingly, these transcription factors have long been known to regulate drought stress/circadian clock (Sanchez et al., 2011), indicating that ABA signaling might be mainly involved in the regulation of leaf degreening-triggered by abiotic stresses. JA signaling directly regulates leaf degreening by MYC2/3/4 and ANAC019/055/072 (Zhu et al., 2015). Considering that the MYCs and ANACs are also involved in the regulation of defense responses, JA signaling likely mediates the degreening process incurred by biotic stresses. Light signal, on the other hand, inhibits leaf degreening by both maintaining the transcription of SOC1 and repressing the transcription of PIFs/reducing PIFs protein accumulation
(Sakuraba et al., 2014; Song et al., 2014; Zhang et al., 2015; Chen et al., 2017). Intriguingly, major hormones share their signaling components with light, as loss-of-function mutations of major hormone signaling components (EIN2, EIN3/EIL1, ABI5, EEL, NAP, ORE1, etc.) block light signaling in regulating degreening, causing stay-green phenotypes upon light deprivation, whereas loss-of-functions of major light signaling components, PIFs, also interfere major hormone (e.g., ethylene) signaling in the promotion of degreening (Oh et al., 1997; Guo and Gan, 2006; Li et al., 2013; Kim et al., 2014; Sakuraba et al., 2014; Song et al., 2014).

Although, substantial progress has been made in exploring the molecular regulation of $\mathrm{Chl}$ degradation, numerous issues still await to be addressed. (1) There appears to be a "developmental window" for hormone-induced Chl degradation. Ethylene, for example, cannot readily induce leaves to degreen at their young age, and only after a certain developmental stage will leaves allow ethylene to induce their degreening (Jing et al., 2005). What is the molecular basis for the "window effect"? (2) As an inhibitor of Chl degradation, light signal is present during ethylene-, ABA-, and JA-induced or age-dependent leaf degreening (Qiu et al., 2015; Zhu et al., 2015; Gao et al., 2016), but how ethylene, ABA, or JA signaling antagonize light signaling to trigger Chl degradation? (3) There are enormous cross-talks among different hormone signaling pathways which are interweaved with light signaling in the regulation of $\mathrm{Chl}$ degradation. It was reported that ein 3 exhibited a stay-green phenotype during MeJA treatment ( $\mathrm{Li}$ et al., 2013), and jaz7 showed an early yellowing phenotype under dark treatment (Yu et al., 2016). More work need to be done to elucidate those cross-talks. (4) In addition to ethylene, $\mathrm{ABA}$, and JA, other phytohormones are also found to be involved in the regulation of $\mathrm{Chl}$ degradation, with salicylic acid and brassinolide acting as promoters (Morris et al., 2000; Jeong et al., 2010), whereas cytokinin and gibberellic acid as repressors (Fletcher and Osborne, 1966; Lara et al., 2004; Kim et al., 2006). Yet, their regulatory pathways or networks are largely unexplored. (4) Thus far, studies on Chl degradation regulation mainly focus on the transcriptional level. Further investigations need to be extended to post-transcriptional levels, including the translational regulation and post-translational modification. It has been reported that PAO could be interconverted between phosphorylated and dephosphorylated status (Chung et al., 2006).

\section{AUTHOR CONTRIBUTIONS}

All authors listed have made a substantial, direct, and intellectual contribution to the work, and approved it for publication.

\section{FUNDING}

This work was supported by National Natural Science Foundation of China $(31670287,31170218$ ) (to BK) and National Postdoctoral Program for Innovative Talents (BX201700058) (to JC). 


\section{REFERENCES}

Armstead, I., Donnison, I., Aubry, S., Harper, J., Hörtensteiner, S., James, C., et al. (2007). Cross-species identification of Mendel's I locus. Science 315:73. doi: $10.1126 /$ science. 1132912

Beltrano, J., Ronco, M. G., Montaldi, E. R., and Carbone, A. (1998). Senescence of flag leaves and ears of wheat hastened by methyl jasmonate. J. Plant Growth Regul. 17, 53-57. doi: 10.1007/PL00007012

Bleecker, A. B., Estelle, M. A., Somerville, C., and Kende, H. (1988). Insensitivity to ethylene conferred by a dominant mutation in Arabidopsis thaliana. Science 241, 1086-1089.

Breeze, E., Harrison, E., McHattie, S., Hughes, L., Hickman, R., Hill, C., et al. (2011). High-resolution temporal profiling of transcripts during Arabidopsis leaf senescence reveals a distinct chronology of processes and regulation. Plant Cell 23, 873-894. doi: 10.1105/tpc.111.083345

Bu, Q., Jiang, H., Li, C.-B., Zhai, Q., Zhang, J., Wu, X., et al. (2008). Role of the Arabidopsis thaliana NAC transcription factors ANAC019 and ANAC055 in regulating jasmonic acid-signaled defense responses. Cell Res. 18, 756-767. doi: $10.1038 / \mathrm{cr} .2008 .53$

Burg, S. P. (1973). Ethylene in plant growth. Proc. Natl. Acad. Sci. U.S.A. 70, 591-597. doi: 10.1073/pnas.70.2.591

Castillo, M. C., and León, J. (2008). Expression of the $\beta$-oxidation gene 3ketoacyl-CoA thiolase 2 (KAT2) is required for the timely onset of natural and dark-induced leaf senescence in Arabidopsis. J. Exp. Bot. 59, 2171-2179. doi: $10.1093 /$ jxb/ern079

Chao, Q., Rothenberg, M., Solano, R., Roman, G., Terzaghi, W., and Ecker, J. R. (1997). Activation of the ethylene gas response pathway in Arabidopsis by the nuclear protein ETHYLENE-INSENSITIVE3 and related proteins. Cell 89, 1133-1144. doi: 10.1016/S0092-8674(00)80300-1

Chen, J., Ren, G., and Kuai, B. (2016). The mystery of Mendel's staygreen: magnesium stays chelated in chlorophylls. Mol. Plant 9, 1556-1558. doi: 10.1016/j.molp.2016.11.004

Chen, J., Zhu, X., Ren, J., Qiu, K., Li, Z., Xie, Z., et al. (2017). Suppressor of overexpression of $\mathrm{CO} 1$ negatively regulates dark-induced leaf degreening and senescence by directly repressing pheophytinase and other senescence-associated genes in Arabidopsis. Plant Physiol. 173, 1881-1891. doi: $10.1104 /$ pp.16.01457

Christ, B., and Hörtensteiner, S. (2014). Mechanism and significance of chlorophyll breakdown. J. Plant Growth Regul. 33, 4-20. doi: 10.1007/s00344-0139392-y

Christ, B., Schelbert, S., Aubry, S., Süssenbacher, I., Müller, T., Kräutler, B., et al. (2012). MES16, a member of the methylesterase protein family, specifically demethylates fluorescent chlorophyll catabolites during chlorophyll breakdown in Arabidopsis. Plant Physiol. 158, 628-641. doi: 10.1104/pp.111.1 88870

Christ, B., Süssenbacher, I., Moser, S., Bichsel, N., Egert, A., Müller, T., et al. (2013). Cytochrome P450 CYP89A9 is involved in the formation of major chlorophyll catabolites during leaf senescence in Arabidopsis. Plant Cell 25, 1868-1880. doi: $10.1105 /$ tpc.113.112151

Chung, D. W., Pružinská, A., Hörtensteiner, S., and Ort, D. R. (2006). The role of pheophorbide a oxygenase expression and activity in the canola green seed problem. Plant Physiol. 142, 88-97. doi: 10.1104/pp.106.08 4483

Delmas, F., Sankaranarayanan, S., Deb, S., Widdup, E., Bournonville, C., Bollier, N., et al. (2013). ABI3 controls embryo degreening through Mendel's I locus. Proc. Natl. Acad. Sci. U.S.A. 110, e3888-e3894. doi: 10.1073/pnas.1308114110

Fletcher, R. A., and Osborne, D. J. (1966). Gibberellin, as a regulator of protein and ribonucleic acid synthesis during senescence in leaf cells of taraxacum officinale. Can. J. Bot. 44, 739-745. doi: 10.1139/b66-088

Gao, S., Gao, J., Zhu, X., Song, Y., Li, Z., Ren, G., et al. (2016). ABF2, $\mathrm{ABF} 3$, and $\mathrm{ABF} 4$ promote $\mathrm{ABA}$-mediated chlorophyll degradation and leaf senescence by transcriptional activation of chlorophyll catabolic genes and senescence-associated genes in Arabidopsis. Mol. Plant 9, 1272-1285. doi: 10.1016/j.molp.2016.06.006

Ghandchi, F. P., Caetano-Anolles, G., Clough, S. J., and Ort, D. R. (2016). Investigating the control of chlorophyll degradation by genomic correlation mining. PLoS ONE 11:e0162327. doi: 10.1371/journal.pone.016 2327
Grbic, V., and Bleecker, A. B. (1995). Ethylene regulates the timing of leaf senescence in Arabidopsis. Plant J. 8, 595-602. doi: 10.1046/j.1365-313X.1995.8040595.x

Guo, Y., and Gan, S. (2006). AtNAP, a NAC family transcription factor, has an important role in leaf senescence. Plant J. 46, 601-612. doi: 10.1111/j.1365-313X.2006.02723.x

Hauenstein, M., Christ, B., Das, A., Aubry, S., and Hörtensteiner, S. (2016). A role for TIC55 as a hydroxylase of phyllobilins, the products of chlorophyll breakdown during plant senescence. Plant Cell 28, 2510-2527. doi: $10.1105 /$ tpc. 16.00630

He, Y., Fukushige, H., Hildebrand, D. F., and Gan, S. (2002). Evidence supporting a role of jasmonic acid in Arabidopsis leaf senescence. Plant Physiol. 128, 876-884. doi: 10.1104/pp.010843

Horie, Y., Ito, H., Kusaba, M., Tanaka, R., and Tanaka, A. (2009). Participation of chlorophyll $b$ reductase in the initial step of the degradation of lightharvesting chlorophyll a/b-protein complexes in Arabidopsis. J. Biol. Chem. 284, 17449-17456. doi: 10.1074/jbc.M109.008912

Hörtensteiner, S. (2006). Chlorophyll degradation during senescence. Annu. Rev. Plant Biol. 57, 55-77. doi: 10.1146/annurev.arplant.57.032905.105212

Jeong, Y. J., Shang, Y., Kim, B. H., Kim, S. Y., Song, J. H., Lee, J. S., et al. (2010). BAK7 displays unequal genetic redundancy with BAK1 in brassinosteroid signaling and early senescence in Arabidopsis. Mol. Cells 29, 259-266. doi: 10.1007/s10059-010-0024-0

Jing, H. C., Schippers, J. H., Hille, J., and Dijkwel, P. P. (2005). Ethylene-induced leaf senescence depends on age-related changes and OLD genes in Arabidopsis. J. Exp. Bot. 56, 2915-2923. doi: 10.1093/jxb/eri287

John, I., Drake, R., Farrell, A., Cooper, W., Lee, P., Horton, P., et al. (1995). Delayed leaf senescence in ethylene-deficient ACC-oxidase antisense tomato plants: molecular and physiological analysis. Plant J. 7, 483-490. doi: 10.1046/j.1365-313X.1995.7030483.X

Kim, H. J., Hong, S. H., Kim, Y. W., Lee, I. H., Jun, J. H., Phee, B. K., et al. (2014). Gene regulatory cascade of senescence-associated NAC transcription factors activated by ETHYLENE-INSENSITIVE2-mediated leaf senescence signalling in Arabidopsis. J. Exp. Bot. 65, 4023-4036. doi: 10.1093/jxb/eru112

Kim, H. J., Ryu, H., Hong, S. H., Woo, H. R., Lim, P. O., Lee, I. C., et al. (2006). Cytokinin-mediated control of leaf longevity by AHK3 through phosphorylation of ARR2 in Arabidopsis. Proc. Natl. Acad. Sci. U.S.A. 103, 814-819. doi: 10.1073/pnas.0505150103

Kim, J. H., Woo, H. R., Kim, J., Lim, P. O., Lee, I. C., Choi, S. H., et al. (2009). Trifurcate feed-forward regulation of age-dependent cell death involving miR164 in Arabidopsis. Science 323, 1053-1057. doi: 10.1126/science.11 66386

Kim, Y. S., Sakuraba, Y., Han, S. H., Yoo, S. C., and Paek, N. C. (2013). Mutation of the Arabidopsis NAC016 transcription factor delays leaf senescence. Plant Cell Physiol. 54, 1660-1672. doi: 10.1093/pcp/pct113

Kou, X., Watkins, C. B., and Gan, S. S. (2012). Arabidopsis AtNAP regulates fruit senescence. J. Exp. Bot. 63, 6139-6147. doi: 10.1093/jxb/ers266

Kusaba, M., Ito, H., Morita, R., Iida, S., Sato, Y., Fujimoto, M., et al. (2007). Rice NON-YELLOW COLORING1 is involved in light-harvesting complex II and grana degradation during leaf senescence. Plant Cell 19, 1362-1375. doi: 10.1105/tpc.106.042911

Lara, B. M. E., Garcia, M-C. G., Fatima, T., Ehneß, R., Lee, T. K., Proels, R. et al. (2004). Extracellular invertase is an essential component of cytokinin-mediated delay of senescence. Plant Cell 16, 1276-1287. doi: 10.1105/tpc.018929

Lee, S. H., Sakuraba, Y., Lee, T., Kim, K. W., An, G., Lee, H. Y., et al. (2015). Mutation of Oryza sativa CORONATINE INSENSITIVE $1 \mathrm{~b}$ (OsCOI1b) delays leaf senescence. J. Integr. Plant Biol. 57, 562-576. doi: 10.1111/jipb.12276

Leivar, P., Monte, E., Oka, Y., Liu, T., Carle, C., Castillon, A., et al. (2008). Multiple phytochrome-interacting bHLH transcription factors repress premature seedling photomorphogenesis in darkness. Curr. Biol. 18, 1815-1823. doi: 10.1016/j.cub.2008.10.058

Li, S., Gao, J., Yao, L., Ren, G., Zhu, X., Gao, S., et al. (2016). The role of ANAC072 in the regulation of chlorophyll degradation during age- and dark-induced leaf senescence. Plant Cell Rep. 35, 1729-1741. doi: 10.1007/s00299-016-1991-1

Li, Z., Peng, J., Wen, X., and Guo, H. (2013). ETHYLENE-INSENSITIVE3 is a senescence-associated gene that accelerates age-dependent leaf senescence by directly repressing miR164 transcription in Arabidopsis. Plant Cell 25, 3311-3328. doi: 10.1105/tpc.113.113340 
Li, Z., Wu, S., Chen, J., Wang, X., Gao, J., Ren, G., et al. (2017). NYEs/SGRsmediated chlorophyll degradation is critical for detoxification during seed maturation in Arabidopsis. Plant J. doi: 10.1111/tpj.13710. [Epub ahead of print].

Liang, C., Wang, Y., Zhu, Y., Tang, J., Hu, B., Liu, L., et al. (2014). OsNAP connects abscisic acid and leaf senescence by fine-tuning abscisic acid biosynthesis and directly targeting senescence-associated genes in rice. Proc. Natl. Acad. Sci. U.S.A. 111, 10013-10018. doi: 10.1073/pnas.1321568111

Lim, P. O., Kim, H. J., and Gil Nam, H. (2007). Leaf senescence. Annu. Rev. Plant Biol. 58, 115-136. doi: 10.1146/annurev.arplant.57.032905.105316

Liu, T., Longhurst, A. D., Talavera-Rauh, F., Hokin, S. A., and Barton, M. K. (2016). The Arabidopsis transcription factor ABIG1 relays ABA signaled growth inhibition and drought induced senescence. Elife 5:e13768. doi: 10.7554/eLife. 13768

Mao, C., Lu, S., Lv, B., Zhang, B., Shen, J., He, J., et al. (2017). A rice NAC transcription factor promotes leaf senescence via ABA biosynthesis. Plant Physiol. 174, 1747-1763. doi: 10.1104/pp.17.00542

Meguro, M., Ito, H., Takabayashi, A., Tanaka, R., and Tanaka, A. (2011). Identification of the 7-hydroxymethyl chlorophyll a reductase of the chlorophyll cycle in Arabidopsis. Plant Cell 23, 3442-3453. doi: 10.1105/tpc.111.089714

Morita, R., Sato, Y., Masuda, Y., Nishimura, M., and Kusaba, M. (2009). Defect in non-yellow coloring 3 , an $\alpha / \beta$ hydrolase-fold family protein, causes a stay-green phenotype during leaf senescence in rice. Plant J. 59, 940-952. doi: $10.1111 / j .1365-313 X .2009 .03919 . x$

Morris, K., MacKerness, S. A., Page, T., John, C. F., Murphy, A. M., and Carr, J. P., et al. (2000). Salicylic acid has a role in regulating gene expression during leaf senescence. Plant J. 23, 677-685. doi: 10.1046/j.1365-313x.2000.00836.x

Niu, F., Wang, C., Yan, J., Guo, X., Wu, F., Yang, B., et al. (2016). Functional characterization of NAC55 transcription factor from oilseed rape (Brassica napus L.) as a novel transcriptional activator modulating reactive oxygen species accumulation and cell death. Plant Mol. Biol. 92, 89-104. doi: $10.1007 / \mathrm{s} 11103-016-0502-7$

Nusinow, D. A., Helfer, A., Hamilton, E. E., King, J. J., Imaizumi, T., Schultz, T. F., et al. (2011). The ELF4-ELF3-LUX complex links the circadian clock to diurnal control of hypocotyl growth. Nature 475, 398-402. doi: 10.1038/nature10182

Oda-Yamamizo, C., Mitsuda, N., Sakamoto, S., Ogawa, D., Ohmetakagi, M., and Ohmiya, A. (2016). Corrigendum: the NAC transcription factor ANAC046 is a positive regulator of chlorophyll degradation and senescence in Arabidopsis leaves. Sci. Rep. 6:23609. doi: 10.1038/srep35125

Oh, S. A., Park, J. H., Lee, G. I., Paek, K. H., Park, S. K., and Nam, H. G. (1997). Identification of three genetic loci controlling leaf senescence in Arabidopsis thaliana. Plant J. 12, 527-535. doi: 10.1046/j.1365-313X.1997.00527.x

Pruzinská, A., Anders, I., Aubry, S., Schenk, N., Tapernoux-Lüthi, E., Müller, T., et al. (2007). In vivo participation of red chlorophyll catabolite reductase in chlorophyll breakdown. Plant Cell 19, 369-387. doi: 10.1105/tpc.106.044404

Pružinská, A., Tanner, G., Anders, I., Roca, M., and Hörtensteiner, S. (2003). Chlorophyll breakdown: pheophorbide a oxygenase is a Rieske-type iron-sulfur protein, encoded by the accelerated cell death 1 gene. Proc. Natl. Acad. Sci. U.S.A. 100, 15259-15264. doi: 10.1073/pnas.2036571100

Qi, T., Wang, J., Huang, H., Liu, B., Gao, H., Liu, Y., et al. (2015). Regulation of jasmonate-induced leaf senescence by antagonism between bHLH subgroup IIIe and IIId factors in Arabidopsis. Plant Cell 27, 1634-1649. doi: 10.1105/tpc.15.00110

Qiu, K., Li, Z., Yang, Z., Chen, J., Wu, S., Zhu, X., et al. (2015). EIN3 and ORE1 accelerate degreening during ethylene-mediated leaf senescence by directly activating chlorophyll catabolic genes in Arabidopsis. PLoS Genet. 11:e1005399. doi: 10.1371/journal.pgen.1005399

Raab, S., Drechsel, G., Zarepour, M., Hartung, W., Koshiba, T., Bittner, F., et al. (2009). Identification of a novel E3 ubiquitin ligase that is required for suppression of premature senescence in Arabidopsis. Plant J. 59, 39-51. doi: 10.1111/j.1365-313X.2009.03846.x

Ren, G., An, K., Liao, Y., Zhou, X., Cao, Y., Zhao, H., et al. (2007). Identification of a novel chloroplast protein AtNYE1 regulating chlorophyll degradation during leaf senescence in Arabidopsis. Plant Physiol. 144, 1429-1441. doi: 10.1104/pp.107.100172

Ren, G., Zhou, Q., Wu, S., Zhang, Y., Zhang, L., Huang, J., et al. (2010). Reverse genetic identification of CRN1 and its distinctive role in chlorophyll degradation in Arabidopsis. J. Integr. Plant Biol. 52, 496-504. doi: 10.1111/j.1744-7909.2010.00945.x

Sakuraba, Y., Han, S. H., Lee, S. H., Hörtensteiner, S., and Paek, N. C. (2016). Arabidopsis NAC016 promotes chlorophyll breakdown by directly upregulating STAYGREEN1 transcription. Plant Cell Rep. 35, 155-166. doi: 10.1007/s00299-015-1876-8

Sakuraba, Y., Jeong, J., Kang, M. Y., Kim, J., Paek, N. C., and Choi, G. (2014). Phytochrome-interacting transcription factors PIF4 and PIF5 induce leaf senescence in Arabidopsis. Nat. Commun. 5:5636. doi: 10.1038/ncomms5636

Sanchez, A., Shin, J., and Davis, S. J. (2011). Abiotic stress and the plant circadian clock. Plant Signal. Behav. 6, 223-231. doi: 10.4161/psb.6.2.14893

Sato, Y., Morita, R., Katsuma, S., Nishimura, M., Tanaka, A., and Kusaba, M. (2009). Two short-chain dehydrogenase/reductases, NON-YELLOW COLORING 1 and NYC1-LIKE, are required for chlorophyll b and lightharvesting complex II degradation during senescence in rice. Plant J. 57, 120-131. doi: 10.1111/j.1365-313X.2008.03670.x

Schäfer, E., and Bowler, C. (2002). Phytochrome-mediated photoperception and signal transduction in higher plants. EMBO Rep. 3, 1042-1048. doi: 10.1093/embo-reports/kvf222

Schelbert, S., Aubry, S., Burla, B., Agne, B., Kessler, F., Krupinska, K., et al. (2009). Pheophytin pheophorbide hydrolase (pheophytinase) is involved in chlorophyll breakdown during leaf senescence in Arabidopsis. Plant Cell 21, 767-785. doi: 10.1105/tpc.108.064089

Schommer, C., Palatnik, J. F., Aggarwal, P., Chételat, A., Cubas, P., Farmer, E. E., et al. (2008). Control of jasmonate biosynthesis and senescence by miR319 targets. PLoS Biol. 6:230. doi: 10.1371/journal.pbio.0060230

Serek, M., Sisler, E. C., and Reid, M. S. (1995). Effects of 1-MCP on the vase life and ethylene response of cut flowers. Plant Growth Regul. 16, 93-97. doi: 10.1007/BF00040512

Shan, X., Wang, J., Chua, L., Jiang, D., Peng, W., and Xie, D. (2011). The role of Arabidopsis rubisco activase in jasmonate-induced leaf senescence. Plant Physiol. 155, 751-764. doi: 10.1104/pp.110.166595

Sheard, L. B., Tan, X., Mao, H., Withers, J., Ben-Nissan, G., Hinds, T. R., et al. (2010). Jasmonate perception by inositol-phosphate-potentiated COI1-JAZ co-receptor. Nature 468, 400-405. doi: 10.1038/nature09430

Shimoda, Y., Ito, H., and Tanaka, A. (2016). Arabidopsis STAY-GREEN, Mendel's green cotyledon gene, encodes magnesium-dechelatase. Plant Cell 28, 2147-2160. doi: 10.1105/tpc.16.00428

Shin, J., Kim, K., Kang, H., Zulfugarov, I. S., Bae, G., Lee, C.-H., et al. (2009). Phytochromes promote seedling light responses by inhibiting four negativelyacting phytochrome-interacting factors. Proc. Natl. Acad. Sci. U.S.A. 106, 7660-7665. doi: 10.1073/pnas.0812219106

Song, Y., Yang, C., Gao, S., Zhang, W., Li, L., and Kuai, B. (2014). Age-triggered and dark-induced leaf senescence require the bHLH transcription factors PIF3, 4, and 5. Mol. Plant 7, 1776-1787. doi: 10.1093/mp/ssu109

Takasaki, H., Maruyama, K., Takahashi, F., Fujita, M., Yoshida, T., Nakashima, K., et al. (2015). SNAC-As, stress-responsive NAC transcription factors, mediate ABA-inducible leaf senescence. Plant J. 84, 1114-1123. doi: 10.1111/ tpj.13067

Tanaka, R., Hirashima, M., Satoh, S., and Tanaka, A. (2003). The Arabidopsisaccelerated cell death gene ACD1 is involved in oxygenation of pheophorbide a: inhibition of the pheophorbide a oxygenase activity does not lead to the stay-green phenotype in Arabidopsis. Plant Cell Physiol. 44, 1266-1274. doi: $10.1093 / \mathrm{pcp} / \mathrm{pcg} 172$

Tsuchisaka, A., Yu, G., Jin, H., Alonso, J. M., Ecker, J. R., Zhang, X., et al. (2009). A combinatorial interplay among the 1-aminocyclopropane-1-carboxylate isoforms regulates ethylene biosynthesis in Arabidopsis thaliana. Genetics 183, 979-1003. doi: 10.1534/genetics.109.107102

Ueda, J., and Kato, J. (1980). Isolation and identification of a senescence-promoting substance from wormwood (Artemisia absinthium L.). Plant Physiol. 66, 246-249. doi: 10.1104/pp.66.2.246

Uji, Y., Akimitsu, K., and Gomi, K. (2017). Identification of OsMYC2regulated senescence-associated genes in rice. Planta 245, 1241-1246. doi: 10.1007/s00425-017-2697-5

van der Graaff, E., Schwacke, R., Schneider, A., Desimone, M., Flügge, U.-I., and Kunze, R. (2006). Transcription analysis of Arabidopsis membrane transporters and hormone pathways during developmental and induced leaf senescence. Plant Physiol. 141, 776-792. doi: 10.1104/pp.106.079293 
Vom Dorp, K., Hölzl, G., Plohmann, C., Eisenhut, M., Abraham, M., Weber, A. P., et al. (2015). Remobilization of phytol from chlorophyll degradation is essential for tocopherol synthesis and growth of Arabidopsis. Plant Cell 27, 2846-2859. doi: $10.1105 /$ tpc. 15.00395

Wasternack, C., and Hause, B. (2013). Jasmonates: Biosynthesis, perception, signal transduction and action in plant stress response, growth and development. An update to the 2007 review in annals of botany. Ann. Bot. 111, 1021-1058. doi: $10.1093 / \mathrm{aob} / \mathrm{mct} 067$

Wu, S., Li, Z., Yang, L., Xie, Z., Chen, J., Zhang, W., et al. (2016). NON-YELLOWING2 (NYE2), a close paralog of NYE1, plays a positive role in chlorophyll degradation in Arabidopsis. Mol. Plant 9, 624-627. doi: 10.1016/j.molp.2015.12.016

Wüthrich, K. L., Bovet, L., Hunziker, P. E., Donnison, I. S., and Hörtensteiner, S. (2000). Molecular cloning, functional expression and characterisation of RCC reductase involved in chlorophyll catabolism. Plant J. 21, 189-198. doi: 10.1046/j.1365-313x.2000.00667.x

Yan, Y., Christensen, S., Isakeit, T., Engelberth, J., Meeley, R., Hayward, A., et al. (2012). Disruption of OPR7 and OPR8 reveals the versatile functions of jasmonic acid in maize development and defense. Plant Cell 24, 1420-1436. doi: 10.1105/tpc.111.094151

Yang, J., Worley, E., and Udvardi, M. (2014). A NAP-AAO3 regulatory module promotes chlorophyll degradation via $\mathrm{ABA}$ biosynthesis in Arabidopsis leaves. Plant Cell 26, 4862-4874. doi: 10.1105/tpc.114.133769

Yang, T. F., Gonzalez-Carranza, Z. H., Maunders, M. J., and Roberts, J. A. (2008). Ethylene and the regulation of senescence processes in transgenic Nicotiana sylvestris plants. Ann. Bot. 101, 301-310. doi: 10.1093/aob/mcm229

Yao, N., and Greenberg, J. T. (2006). Arabidopsis ACCELERATED CELL DEATH2 modulates programmed cell death. Plant Cell 18, 397-411. doi: $10.1105 /$ tpc. 105.036251

Yin, X. R., Xie, X. L., Xia, X. J., Yu, J. Q., Ferguson, I. B., Giovannoni, J. J., et al. (2016). Involvement of an ethylene response factor in chlorophyll degradation during citrus fruit degreening. Plant J. 86, 403-412. doi: 10.1111/tpj.13178
Yu, J., Zhang, Y., Di, C., Zhang, Q., Zhang, K., Wang, C., et al. (2016). JAZ7 negatively regulates dark-induced leaf senescence in Arabidopsis. J. Exp. Bot. 67, 751-762. doi: 10.1093/jxb/erv487

Zhang, K., and Gan, S.-S. (2012). An abscisic acid-AtNAP transcription factor-SAG113 protein phosphatase 2C regulatory chain for controlling dehydration in senescing Arabidopsis leaves. Plant Physiol. 158, 961-969. doi: 10.1104/pp.111.190876

Zhang, Y., Liu, Z., Chen, Y., He, J. X., and Bi, Y. (2015). PHYTOCHROMEINTERACTING FACTOR 5 (PIF5) positively regulates dark-induced senescence and chlorophyll degradation in Arabidopsis. Plant Sci. 237, 57-68. doi: $10.1016 /$ j.plantsci.2015.05.010

Zheng, X. Y., Spivey, N. W., Zeng, W., Liu, P. P., Fu, Z. Q., Klessig, D. F., et al. (2012). Coronatine promotes pseudomonas syringae virulence in plants by activating a signaling cascade that inhibits salicylic acid accumulation. Cell Host Microbe 11, 587-596. doi: 10.1016/j.chom.2012. 04.014

Zhu, X., Chen, J., Xie, Z., Gao, J., Ren, G., Gao, S., et al. (2015). Jasmonic acid promotes degreening via MYC2/3/4- and ANAC019/055/072-mediated regulation of major chlorophyll catabolic genes. Plant J. 84, 597-610. doi: $10.1111 /$ tpj. 13030

Conflict of Interest Statement: The authors declare that the research was conducted in the absence of any commercial or financial relationships that could be construed as a potential conflict of interest.

Copyright (c) $2017 \mathrm{Zhu}$, Chen, Qiu and Kuai. This is an open-access article distributed under the terms of the Creative Commons Attribution License (CC $B Y)$. The use, distribution or reproduction in other forums is permitted, provided the original author(s) or licensor are credited and that the original publication in this journal is cited, in accordance with accepted academic practice. No use, distribution or reproduction is permitted which does not comply with these terms. 